\title{
IMPACT OF SERUM 25(OH) VITAMIN D LEVEL ON MORTALITY IN PATIENTS WITH COVID-19 IN TURKEY
}

\author{
S. KARAHAN, F. KATKAT \\ Bagcilar Egitim ve Arastirma Hastanesi, Turkey. Corresponding author: Serkan Karahan, Bagcilar Egitim ve Arastirma Hastanesi, Turkey, drserkankarahan@gmail.com
}

\begin{abstract}
Background: Because of the lack of sufficient data, we aimed to investigate the role of serum $25(\mathrm{OH})$ vitamin D level on COVID severity and related mortality. Methods: This was a retrospective observational study. Data, including sociodemographic features, clinical characteristics, and laboratory data, and 25(OH) vitamin D levels were recorded for each study participant. Patients were stratified into different vitamin D groups; Normal (Serum 25(OH) vitamin D level $>30 \mathrm{ng} / \mathrm{mL})$, Vitamin D insufficiency $(21-29 \mathrm{ng} / \mathrm{mL})$, and deficiency $(<20 \mathrm{ng} /$ $\mathrm{mL}$ ). The severity of COVID was classified according to the Chinese Clinical Guideline for classification of COVID-19 severity. Mortality data were determined for participants. Univariate and multivariate Logistic regression analysis was performed to determine independent predictors of in-hospital mortality. Results: Overall, 149 COVID-19 patients (females 45.6\%, mean age $63.5 \pm 15.3$ (range 24-90 years) years) were included. Fortyseven patients $(31.5 \%)$ had moderate COVID-19, whereas 102 patients $(68.5 \%)$ had severe-critical COVID-19. The mean $25(\mathrm{OH})$ vitamin D level was $15.2 \pm 10.3 \mathrm{ng} / \mathrm{mL}$. Thirty-four $(22.8 \%)$ and $103(69.1 \%)$ patients had vitamin D insufficiency and deficiency, respectively. Mean serum $25(\mathrm{OH})$ vitamin D level was significantly lower in patients with severe-critical COVID-19 compared with moderate COVID-19 (10.1 \pm 6.2 vs. $26.3 \pm 8.4$ $\mathrm{ng} / \mathrm{mL}$, respectively, $\mathrm{p}<0.001)$. Vitamin D insufficiency was present in $93.1 \%$ of the patients with severe-critical COVID-19. Multivariate logistic regression analysis revealed that only lymphocyte count, white blood cell count, serum albumin and, 25(OH) vitamin D level were independent predictors of mortality. Conclusion: Serum $25(\mathrm{OH})$ vitamin D was independently associated with mortality in COVID-19 patients.
\end{abstract}

Key words: COVID-19, mortality, prognosis, vitamin D.

\section{Introduction}

Coronavirus disease 2019 (COVID-19) has become a pandemic and claimed more than 400.000 lives throughout the world as the time of the writing of this manuscript (1). Advanced age and underlying chronic medical conditions, particularly chronic pulmonary and cardiac diseases, appeared as the most conspicuous predisposing factors for the development of severe COVID-19 and consequent death $(2,3)$. However, recent observations regarding the relatively greater impact of the disease on the northern latitudes led to the generation of the hypothesis that vitamin D deficiency, to some extent, might have caused a tendency for an increased burden of the disease in vitamin D deficient COVID-19 patients, especially among the elderly (4-6).

Vitamin D is a multi-faceted hormone, the best-known effect of which is on the calcium and phosphorus metabolism. However, multiple lines of experimental and clinical evidence point to its essential regulatory role in both the innate and adaptive immune systems (7). Through several different but additive mechanisms, vitamin D produces anti-viral effects (8). Although not all included studies supported its conclusion, a meta-analysis found a protective effect of vitamin D supplementation against upper respiratory tract viral infections (9). First reports with respect to the role of vitamin D serum levels on contraction of SARS-CoV2 revealed that patients with PCR-proven COVID-19 had significantly lower serum 25(OH) D levels compared with patients without COVID-19, even after controlling for the effect of age (10). However, a larger study did not confirm the results of this latter study (11).

To the best of our knowledge, despite the extensive discussion of the role of vitamin D in COVID-19 susceptibility and development of severe disease through editorials and review articles, only one study evaluated the effect of vitamin D status on mortality in COVID-19 patients. Raharusun and colleagues (12) reported that in 780 patients with laboratoryconfirmed COVID-19 in Indonesia, vitamin D status was an independent predictor of mortality. Considering the ongoing high death toll of COVID-19 along with the prevalent nature of vitamin $\mathrm{D}$ deficiency, we think that it is of utmost importance to delineate the role of vitamin $\mathrm{D}$ status on the prognosis of patients with COVID-19.

Hence, the primary objective of this study was to evaluate the association of vitamin D status with disease severity and mortality in patients with COVID-19.

\section{Methods}

\section{Patients, study design and data collection}

This was a retrospective observational study that included adult COVID-19 patients in Health Sciences University, Bagcilar Training and Research Hospital, Istanbul, Turkey between April 1st, 2020 and May 20th, 2020. The study protocol was approved by the Hospital Clinical Studies Ethical Committee (2020.06.1.01.072 and 12 June 2020).

The primary outcome measure of the study was all-cause 
mortality in COVID-19 patients.

Data, including sociodemographic features, clinical characteristics, and laboratory data comprising complete blood counts, serum albumin, calcium, and $25(\mathrm{OH})$ vitamin D levels were retrieved from the hospital electronic database system and recorded for each study participant.

Patients who did not have $25(\mathrm{OH})$ vitamin $\mathrm{D}$ values, who had clinical presentation compatible with COVID-19, but did not have a positive PCR-based test for SARS-CoV2, and pediatric patients were excluded from the study.

\section{Measurement of serum 25(OH) vitamin D level and Vitamin D groups}

Serum $25(\mathrm{OH})$ vitamin D levels were studied by electrochemiluminescence method. Patients were stratified into different groups according to their serum $25(\mathrm{OH})$ vitamin D levels. Serum $25(\mathrm{OH})$ vitamin D level $>30 \mathrm{ng} / \mathrm{mL}$ was accepted as normal. Vitamin D insufficiency and deficiency were defined as serum $25(\mathrm{OH})$ vitamin D levels of $21-29 \mathrm{ng} / \mathrm{mL}$ and $<20 \mathrm{ng} /$ $\mathrm{mL}$, respectively (13).

\section{Classification of the severity of COVID-19}

We used the Chinese Clinical Guideline for classification of COVID-19 severity, which was devised by the China Health Committee (14). According to this guideline, COVID-19 has four levels of the severity: mild, moderate, severe, and critical. Patient symptoms, laboratory values, and results of imaging studies performed at admission are used to determine severity of COVID-19. Based on this classification system, disease severity groups were defined as follows;

- Mild disease: Mild clinical symptoms and normal lung on radiologic imaging

- Moderate disease: Fever and pulmonary symptoms along with pneumonia on radiologic imaging

- Severe disease: The presence of any of the following criteria: i) respiratory distress ( $\geq 30$ breaths $/ \mathrm{min}$ ); ii) oxygen saturation $\leq 93 \%$ at rest; iii) $\mathrm{PaO} 2 / \mathrm{FiO} 2 \leq 300 \mathrm{mmHg}$ or chest imaging shows obvious lesion progression $>50 \%$ within 24-48 hours)

- Critical disease: The presence of any of the following criteria: i) respiratory failure and need for mechanical ventilation; ii) shock; iii) other organ failures that requires ICU care.

Since patients with mild COVID-19 were not hospitalized according to our National guidelines, our study did not involve any patient with mild disease. Furthermore, for practical purposes for our study aims, we combined severe and critical COVID-19 in a single group named "severe-critical disease". In the end, we stratified all included patients either to moderate or critical-severe COVID-19 groups.

\section{Statistical analysis}

In summarization of the study data, descriptive statistics were presented for continuous variables as either mean +/standard deviation or median-interquartile range depending on the distribution type of the data. Categorical variables were reported as numbers and percentages. The normality check of numerical variables was performed via the Kolmogorov-Smirnov test. According to groups, to compare categoric variables, chi-square/Fisher exact test used, while Independent Samples t-test or Mann-Whitney U tests was used for continuous variables in case of normal and non-normal distribution, respectively. To evaluate the bivariate correlation between the serum $25(\mathrm{OH})$ vitamin D level and inflammatory marker, Pearson's correlation was used. We also constructed univariate and multivariate logistic regression analyses to determine the independent associates of mortality. SPSS 26.0 (IBM Corporation, NY, US) was used to perform all statistical analyses. A P-value $<0.05$ was considered as statistically significant.

\section{Results}

\section{Patient characteristics and baseline laboratory values}

Overall, 149 COVID-19 patients (females 45.6\%) were included. The mean age of the patients was $63.5 \pm 15.3$ (range 24-90 years) years. The most frequent comorbid condition was hypertension, which was followed by diabetes mellitus (40.9\%) and dyslipidemia (26.2\%). Almost one-fifth of the whole group had chronic kidney disease, and the mean estimated glomerular filtration rate (eGFR) was $74.7 \pm 33.7 \mathrm{~mL} /$ minute.

Sixty-two patients (41.6\%) had critical COVID-19, whereas forty patients had severe disease. Baseline laboratory values of the entire study cohort were shown in table-1.

\section{Moderate and severe-critical COVID-19}

Forty-seven patients $(31.5 \%)$ had moderate COVID-19, whereas 102 patients $(68.5 \%)$ had severe-critical COVID19. Patients with severe-critical COVID-19 had significantly higher rates of coronary artery disease, hypertension, dyslipidemia, diabetes mellitus, chronic kidney disease, chronic atrial fibrillation, and congestive heart failure compared to patients who had moderate COVID-19. Interestingly, chronic obstructive pulmonary disease frequency was comparable between the groups. Patients with severe-critical COVID19 had significantly lower eGFR, lower hemoglobin, higher neutrophil and lymphocyte counts, and higher serum C-reactive protein values compared to those of patients with moderate COVID-19. Table-2 summarizes the comorbid disease rates and laboratory values in moderate and severe-critical COVID19 patients.

\section{5(OH) Vitamin D levels and vitamin D status}

The mean $25(\mathrm{OH})$ vitamin D level was $15.2 \pm 10.3 \mathrm{ng} / \mathrm{mL}$. The numbers of patients with vitamin D insufficiency and 
Table 1

Mean age, sex distribution, comorbid conditions, baseline laboratory values and serum 25(OH) vitamin D levels in the whole study cohort

\begin{tabular}{|c|c|c|c|c|c|}
\hline & & \multicolumn{2}{|c|}{ Min-Max } & \multirow{2}{*}{$\frac{\text { Median }}{65.0}$} & \multirow{2}{*}{$\frac{\text { Mean } \pm \text { SD } / \text { Number }(\%)}{63.5 \pm 15.3}$} \\
\hline Age (years) & & 24.0 & -90.0 & & \\
\hline \multirow[t]{2}{*}{ Sex } & Female & & & & $68(45.6 \%)$ \\
\hline & Male & & & & $81(54.4 \%)$ \\
\hline Smoking & & & & & $51(34.2 \%)$ \\
\hline Coronary artery disease & & & & & $32(21.5 \%)$ \\
\hline Hypertension & & & & & $85(57.0 \%)$ \\
\hline Dyslipidemia & & & & & $39(26.2 \%)$ \\
\hline Diabetes mellitus & & & & & $61(40.9 \%)$ \\
\hline Cerebrovascular accident & & & & & $9(6.0 \%)$ \\
\hline Chronic obstructive pulmonary disease & & & & & $15(10.1 \%)$ \\
\hline Malignancy & & & & & $23(15.4 \%)$ \\
\hline Chronic kidney disease & & & & & $29(19.5 \%)$ \\
\hline Chronic atrial fibrillation & & & & & $15(10.1 \%)$ \\
\hline Congestive heart failure & & & & & $18(12.1 \%)$ \\
\hline Acute kidney injury & & & & & $16(10.7 \%)$ \\
\hline eGFR (mL/minute) & & 3.0 & -141.0 & 81.0 & $74.7 \pm 33.7$ \\
\hline Hemoglobin (g/dL) & & 5.0 & -17.0 & 12.1 & $12.0 \pm 2.1$ \\
\hline Neutrophil count $\left(\times 10^{3} / \mathrm{mm}^{3}\right)$ & & 0.50 & -31.20 & 5.60 & $6.82 \pm 4.84$ \\
\hline Lymphocyte count $\left(\mathrm{x} 10^{3} / \mathrm{mm}^{3}\right)$ & & 0.32 & -27.20 & 1.78 & $4.08 \pm 4.69$ \\
\hline White blood cell count $\left(\times 10^{3} / \mathrm{mm}^{3}\right)$ & & 0.20 & -21.10 & 4.09 & $4.23 \pm 3.37$ \\
\hline Platelet count (x103/mm3) & & 46.0 & -688.0 & 208.0 & $227.3 \pm 96.1$ \\
\hline C-reactive protein $(\mathrm{mg} / \mathrm{dL})$ & & 0.5 & -317.3 & 55.0 & $83.8 \pm 77.8$ \\
\hline Albumin (g/dL) & & 1.9 & -4.6 & 3.3 & $3.3 \pm 0.5$ \\
\hline Calcium (mg/dL) & & 6.3 & -9.6 & 8.2 & $8.2 \pm 0.6$ \\
\hline $25(\mathrm{OH})$ vitamin $\mathrm{D}(\mathrm{ng} / \mathrm{mL})$ & & 1.6 & -57.1 & 11.7 & $15.2 \pm 10.3$ \\
\hline \multirow[t]{3}{*}{ Vitamin D status } & $\leq 20$ (Deficiency) & & & & $103(69.1 \%)$ \\
\hline & 21-29 (Insufficiency) & & & & $34(22.8 \%)$ \\
\hline & $\geq 30$ (Normal) & & & & $12(8.1 \%)$ \\
\hline \multirow[t]{3}{*}{ COVID-19 severity } & Moderate & & & & $47(31.5 \%)$ \\
\hline & Severe & & & & $40(26.8 \%)$ \\
\hline & Critical & & & & $62(41.6 \%)$ \\
\hline
\end{tabular}

deficiency were $34(22.8 \%)$ and 103 (69.1\%), respectively. Only $8.1 \%$ of the entire study cohort had a normal level of serum $25(\mathrm{OH})$ vitamin $\mathrm{D}$ (table-1).

Mean serum 25(OH) vitamin D level was significantly lower in patients with severe-critical COVID-19 compared to that of patients with moderate COVID-19 (10.1 \pm 6.2 vs. $26.3 \pm 8.4$ $\mathrm{ng} / \mathrm{mL}$, respectively, $\mathrm{p}<0.001)$. Vitamin $\mathrm{D}$ insufficiency was present in $93.1 \%$ of the patients with severe-critical COVID-19 (table-2, figure-1).

Mean serum 25(OH) vitamin D level was significantly lower among deceased patients compared with the surviving patients
$(10.4 \pm 6.4$ vs. $19.3 \pm 11.2 \mathrm{ng} / \mathrm{mL}$, respectively, $\mathrm{P}<0.001) . \mathrm{A}$ significantly higher ratio of the deceased patients had vitamin $\mathrm{D}$ deficiency compared with surviving patients $(92.8 \%$ vs. $48.8 \%$, $\mathrm{P}<0.001$ ) (table-3, figure-2).

Mean serum 25(OH) vitamin D level was negatively correlated with increasing age $(\mathrm{r}=-0.3, \mathrm{p}<0.001)$.

Correlation of $25(\mathrm{OH})$ vitamin $\mathrm{D}$ and inflammatory markers

Mean serum 25(OH) vitamin D level was significantly and negatively correlated with serum $\mathrm{C}$-reactive protein level $(\mathrm{r}=-0.253, \mathrm{P}=0.002)$. Mean serum 25(OH) vitamin $\mathrm{D}$ level was significantly and negatively correlated with neutrophil 
Table 2

Comparison of age, sex distributions, frequency of comorbid conditions, laboratory values and $25(\mathrm{OH})$ vitamin D levels between the COVID-19 patients with moderate and severe-critical disease

\begin{tabular}{|c|c|c|c|c|c|c|c|}
\hline \multirow[b]{3}{*}{ Age } & & \multicolumn{2}{|c|}{ Moderate COVID-19 $(n=47)$} & \multicolumn{2}{|c|}{ Severe-Critical COVID-19 (n=102) } & \multirow{2}{*}{\multicolumn{2}{|c|}{ P-value }} \\
\hline & & Mean \pm SD $/ \mathbf{n}(\%)$ & Median & Mean $\pm \mathrm{SD} / \mathrm{n}(\%)$ & Median & & \\
\hline & & $56.1 \pm 15.2$ & 55.0 & $67.0 \pm 14.1$ & 69.0 & $<0.001$ & $\mathrm{t}$ \\
\hline \multirow[t]{2}{*}{ Sex } & Female & $24(51.1 \%)$ & & $44(43.1 \%)$ & & 0.367 & $\mathrm{X}^{2}$ \\
\hline & Male & $23(48.9 \%)$ & & $58(56.9 \%)$ & & & \\
\hline Smoking & & $10(21.3 \%)$ & & $41(40.2 \%)$ & & 0.024 & $\mathrm{X}^{2}$ \\
\hline Coronary artery disease & & $3(6.4 \%)$ & & $29(28.4 \%)$ & & 0.002 & $\mathrm{X}^{2}$ \\
\hline Hypertension & & $15(31.9 \%)$ & & $70(68.6 \%)$ & & $<0.001$ & $\mathrm{X}^{2}$ \\
\hline Dyslipidemia & & $3(6.4 \%)$ & & $36(35.3 \%)$ & & $<0.001$ & $\mathrm{X}^{2}$ \\
\hline Diabetes mellitus & & $12(25.5 \%)$ & & $49(48.0 \%)$ & & 0.009 & $\mathrm{X}^{2}$ \\
\hline Cerebrovascular accident & & $2(4.3 \%)$ & & $7(6.9 \%)$ & & 0.535 & $\mathrm{X}^{2}$ \\
\hline Chronic obstructive pulmonary disease & & $4(8.5 \%)$ & & $11(10.8 \%)$ & & 0.668 & $\mathrm{X}^{2}$ \\
\hline Malignancy & & $6(12.8 \%)$ & & $17(16.7 \%)$ & & 0.540 & $\mathrm{X}^{2}$ \\
\hline Chronic kidney disease & & $2(4.3 \%)$ & & $27(26.5 \%)$ & & 0.001 & $\mathrm{X}^{2}$ \\
\hline Chronic atrial fibrillation & & $0(0.0 \%)$ & & $15(14.7 \%)$ & & 0.006 & $\mathrm{X}^{2}$ \\
\hline Congestive heart failure & & $0(0.0 \%)$ & & $18(17.6 \%)$ & & 0.002 & $\mathrm{X}^{2}$ \\
\hline Acute kidney injury & & $1(2.1 \%)$ & & $15(14.7 \%)$ & & 0.021 & $\mathrm{X}^{2}$ \\
\hline eGFR & & $94.4 \pm 24.7$ & 101.0 & $65.6 \pm 33.5$ & 71.0 & $<0.001$ & $\mathrm{~m}$ \\
\hline Hemoglobin & & $12.7 \pm 2.0$ & 13.0 & $11.7 \pm 2.1$ & 12.0 & 0.005 & $\mathrm{~m}$ \\
\hline Neutrophil count & & $3.6 \pm 1.8$ & 3.4 & $8.3 \pm 5.1$ & 7.2 & $<0.001$ & $\mathrm{~m}$ \\
\hline Lymphocyte count & & $1.4 \pm 0.6$ & 1.3 & $5.3 \pm 5.2$ & 3.9 & $<0.001$ & $\mathrm{~m}$ \\
\hline White blood cell count & & $5.5 \pm 2.0$ & 5.4 & $3.6 \pm 3.7$ & 1.7 & $<0.001$ & $\mathrm{~m}$ \\
\hline Platelet count & & $219.3 \pm 96.8$ & 199.0 & $231.0 \pm 96.1$ & 212.0 & 0.476 & $\mathrm{~m}$ \\
\hline C-reactive protein & & $44.2 \pm 59.5$ & 17.5 & $102.1 \pm 78.7$ & 83.1 & $<0.001$ & $\mathrm{~m}$ \\
\hline Albumin & & $3.6 \pm 0.5$ & 3.6 & $3.2 \pm 0.5$ & 3.1 & $<0.001$ & $\mathrm{t}$ \\
\hline Calcium & & $8.6 \pm 0.5$ & 8.6 & $8.1 \pm 0.6$ & 8.0 & $<0.001$ & $\mathrm{t}$ \\
\hline $25(\mathrm{OH})$ vitamin $\mathrm{D}$ & & $26.3 \pm 8.4$ & 25.8 & $10.1 \pm 6.2$ & 8.4 & $<0.001$ & $\mathrm{~m}$ \\
\hline \multirow[t]{3}{*}{ Vitamin D status } & $\leq 20$ & 8 & $17.0 \%$ & 95 & $93.1 \%$ & & \\
\hline & $21-29$ & 27 & $57.4 \%$ & 7 & $6.9 \%$ & $<0.001$ & $\mathrm{X}^{2}$ \\
\hline & $\geq 30$ & 12 & $25.5 \%$ & 0 & $0.0 \%$ & & \\
\hline \multirow[t]{2}{*}{ Death } & $(-)$ & 46 & $97.9 \%$ & 34 & $33.3 \%$ & $<0.001$ & $\mathrm{X}^{2}$ \\
\hline & $(+)$ & 1 & $2.1 \%$ & 68 & $66.7 \%$ & & \\
\hline
\end{tabular}

m. Mann-Whitney U test / $\mathrm{t}$ Independent Samples t-test/ $\mathrm{X}^{2}$ Chi-Squared test

count $(\mathrm{r}=-0.419, \mathrm{P}<0.001)$ and lymphocyte count $(\mathrm{r}=-0.348$, $\mathrm{P}<0.001)$.

\section{Mortality and its determinants}

In total, 69 out of 149 patients (46.3\%) died during the study period. The deceased patients were significantly older compared with surviving patients. As expected, the mortality rate was significantly higher among patients with severe-critical COVID-19 compared with moderate COVID-19 patients $(66.7 \%$ vs. $2.1 \%$, respectively, $\mathrm{P}<0.001)$ (Figure-3). 
Figure 1

Box and whisker graph showing $25(\mathrm{OH})$ vitamin $\mathrm{D}$ levels in patients with moderate and severe-critical COVID-19

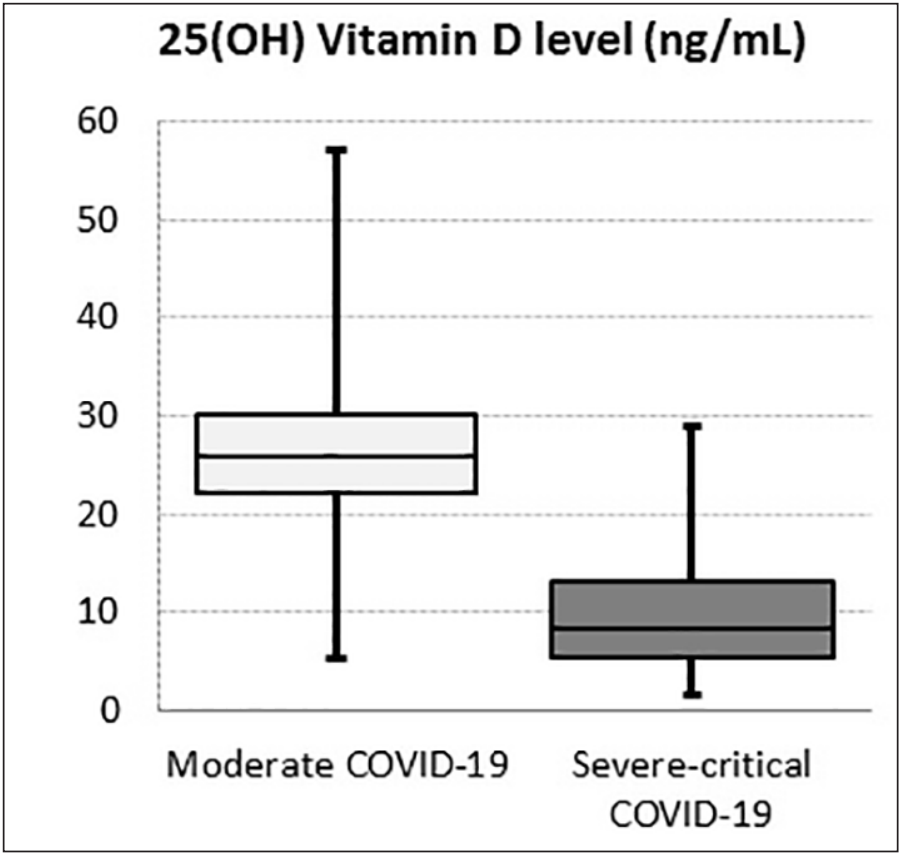

Figure 2

Box and whisker graph showing $25(\mathrm{OH})$ vitamin D levels in the surviving and deceased patients

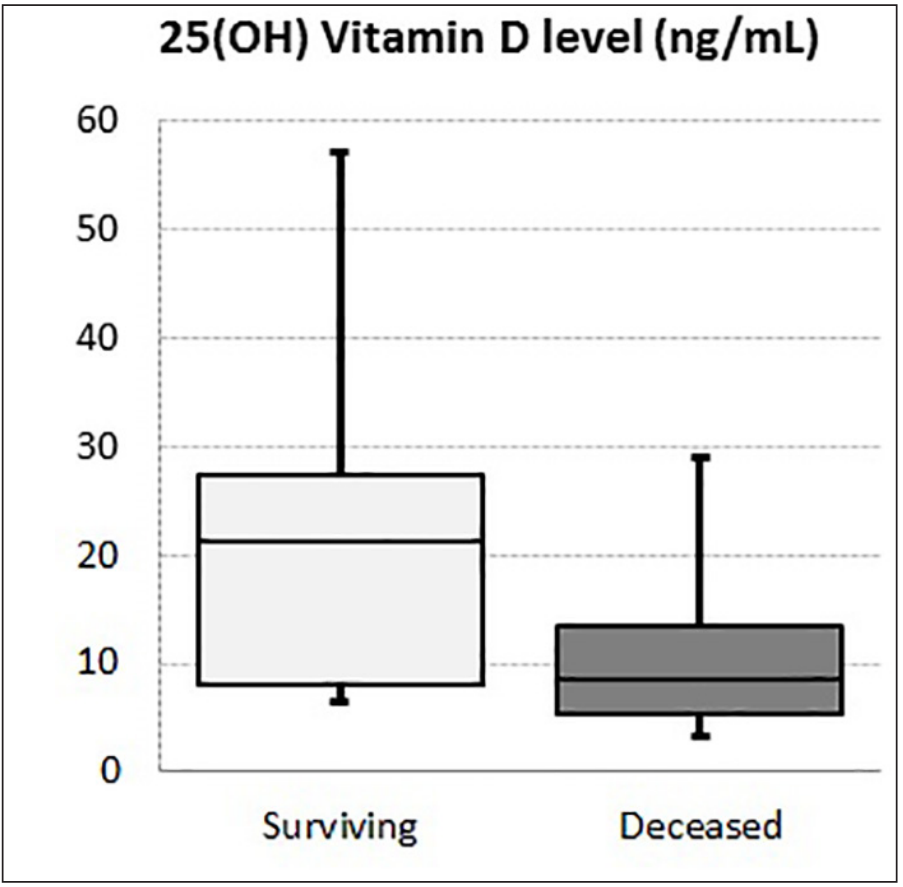

The deceased COVID-19 patients had significantly higher rates of dyslipidemia, diabetes mellitus, chronic kidney disease, chronic atrial fibrillation, and congestive heart failure compared with surviving patients. The deceased COVID-19 patients had significantly lower eGFR, lower hemoglobin, higher neutrophil and lymphocyte counts, and higher C-reactive protein values compared with their surviving counterparts. The comorbid conditions and laboratory results of the deceased and surviving patients were shown in table- 3 .

\section{Figure 3}

Multiple bar chart showing mortality rates in patients with moderate and severe-critical COVID-19

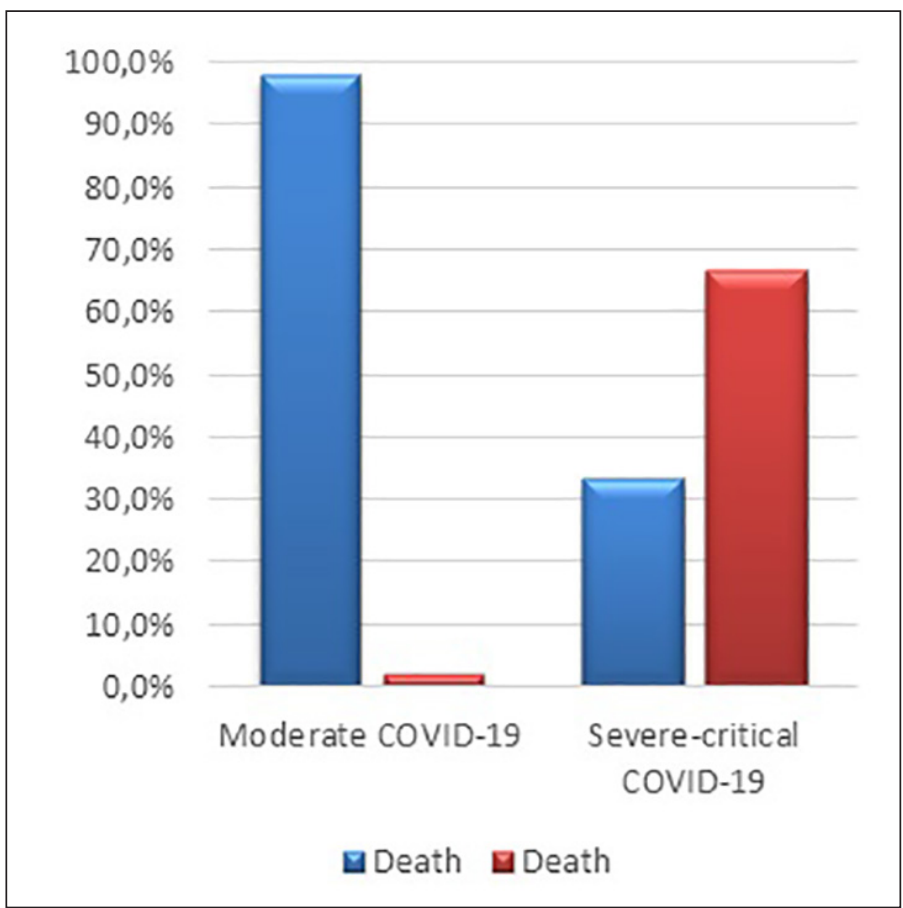

Multivariate logistic regression analysis revealed that only lymphocyte count, white blood cell count, serum albumin, and $25(\mathrm{OH})$ vitamin $\mathrm{D}$ level were independent predictors of mortality. An increase in lymphocyte count, but a decrease in white blood cell count, serum albumin, and $25(\mathrm{OH})$ vitamin D level was associated with increased mortality in COVID-19 patients (table-4).

\section{Discussion}

The salient findings of the present study were as follows: (i) Vitamin D insufficiency (22.8\%) and deficiency $(69.1 \%)$ were quite common among patients with COVID-19. Only $8.1 \%$ of the entire study cohort had a normal level of serum $25(\mathrm{OH})$ vitamin D. (ii) Patients with more severe COVID19 had lower mean serum $25(\mathrm{OH})$ vitamin $\mathrm{D}$ levels. (iii) $\mathrm{A}$ significantly higher ratio of the deceased patients had vitamin $\mathrm{D}$ deficiency compared with surviving patients $(92.8 \%$ vs. $48.8 \%, \mathrm{P}<0.001)$. (iv) Serum $25(\mathrm{OH})$ vitamin $\mathrm{D}$ level was independently associated with increased mortality in COVID19 patients in multivariate logistic regression. 
Table 3

Comparison of age, sex distributions, frequency of comorbid conditions, laboratory values and $25(\mathrm{OH})$ vitamin D levels between the deceased and surviving COVID-19 patients

\begin{tabular}{|c|c|c|c|c|c|c|c|}
\hline & & \multicolumn{2}{|c|}{ Surviving patients } & \multicolumn{2}{|l|}{ Deceased patients } & \multicolumn{2}{|c|}{ P-value } \\
\hline & & Mean \pm SD $/ \mathbf{n}(\%)$ & Median & Mean \pm SD $/ \mathbf{n}(\%)$ & Median & & \\
\hline Age & & $60.0 \pm 15.5$ & 60.0 & $67.7 \pm 14.1$ & 68.5 & 0.002 & $\mathrm{t}$ \\
\hline \multirow[t]{2}{*}{ Sex } & Female & $40(50.0 \%)$ & & \multicolumn{2}{|l|}{$28(40.6 \%)$} & 0.250 & $\mathrm{X}^{2}$ \\
\hline & Male & \multicolumn{2}{|l|}{$40(50.0 \%)$} & \multicolumn{2}{|l|}{$41(59.4 \%)$} & & \\
\hline Smoking & & \multicolumn{2}{|l|}{$21(26.3 \%)$} & \multicolumn{2}{|l|}{$30(43.5 \%)$} & 0.027 & $\mathrm{X}^{2}$ \\
\hline Coronary artery disease & & \multicolumn{2}{|l|}{$13(16.3 \%)$} & \multicolumn{2}{|l|}{$19(27.5 \%)$} & 0.124 & $\mathrm{X}^{2}$ \\
\hline Hypertension & & \multicolumn{2}{|l|}{$41(51.3 \%)$} & \multicolumn{2}{|l|}{$44(63.8 \%)$} & 0.078 & $X^{2}$ \\
\hline Dyslipidemia & & \multicolumn{2}{|l|}{$13(16.3 \%)$} & \multicolumn{2}{|l|}{$26(37.7 \%)$} & 0.003 & $\mathrm{X}^{2}$ \\
\hline Diabetes mellitus & & \multicolumn{2}{|l|}{$25(31.3 \%)$} & \multicolumn{2}{|l|}{$36(52.2 \%)$} & 0.010 & $X^{2}$ \\
\hline Cerebrovascular accident & & \multicolumn{2}{|l|}{$2(2.5 \%)$} & \multicolumn{2}{|l|}{$7(10.1 \%)$} & 0.051 & $\mathrm{X}^{2}$ \\
\hline Chronic obstructive pulmonary disease & & \multicolumn{2}{|l|}{$8(10.0 \%)$} & \multicolumn{2}{|l|}{$7(10.1 \%)$} & 0.977 & $\mathrm{X}^{2}$ \\
\hline Malignancy & & \multicolumn{2}{|l|}{$9(11.3 \%)$} & \multicolumn{2}{|l|}{$14(20.3 \%)$} & 0.128 & $\mathrm{X}^{2}$ \\
\hline Chronic kidney disease & & \multicolumn{2}{|l|}{$9(11.3 \%)$} & \multicolumn{2}{|l|}{$20(29.0 \%)$} & 0.006 & $X^{2}$ \\
\hline Chronic atrial fibrillation & & \multicolumn{2}{|l|}{$2(2.5 \%)$} & \multicolumn{2}{|l|}{$13(18.8 \%)$} & 0.001 & $X^{2}$ \\
\hline Congestive heart failure & & \multicolumn{2}{|l|}{$4(5.0 \%)$} & \multicolumn{2}{|l|}{$14(20.3 \%)$} & 0.004 & $\mathrm{X}^{2}$ \\
\hline Acute kidney injury & & \multicolumn{2}{|l|}{$4(5.0 \%)$} & $12(17.4 \%)$ & & 0.015 & $\mathrm{X}^{2}$ \\
\hline eGFR & & $85.1 \pm 31.8$ & 94.5 & $62.7 \pm 32.0$ & 70.0 & $<0.001$ & $\mathrm{~m}$ \\
\hline Hemoglobin & & $12.4 \pm 1.9$ & 12.5 & $11.6 \pm 2.2$ & 12.0 & 0.027 & $\mathrm{~m}$ \\
\hline Neutrophil count & & $4.7 \pm 3.1$ & 4.1 & $9.3 \pm 5.3$ & 7.8 & $<0.001$ & $\mathrm{~m}$ \\
\hline Lymphocyte count & & $1.7 \pm 2.0$ & 1.2 & $6.8 \pm 5.4$ & 5.4 & $<0.001$ & $\mathrm{~m}$ \\
\hline White blood cell count & & $5.8 \pm 2.9$ & 5.5 & $2.4 \pm 2.9$ & 1.0 & $<0.001$ & $\mathrm{~m}$ \\
\hline Platelet count & & $224.3 \pm 92.9$ & 205.0 & $230.7 \pm 100.3$ & 211.0 & 0.771 & $\mathrm{~m}$ \\
\hline C-reactive protein & & $62.4 \pm 71.2$ & 31.3 & $108.7 \pm 78.3$ & 90.0 & $<0.001$ & $\mathrm{~m}$ \\
\hline Albumin & & $3.5 \mathrm{~N} \pm 0.5$ & 3.5 & $3.1 \pm 0.5$ & 3.0 & $<0.001$ & $\mathrm{t}$ \\
\hline Calcium & & $8.4 \pm 0.6$ & 8.5 & $8.0 \pm 0.6$ & 8.0 & $<0.001$ & $\mathrm{t}$ \\
\hline $25(\mathrm{OH})$ vitamin $\mathrm{D}$ & & $19.3 \pm 11.2$ & 21.3 & $10.4 \pm 6.4$ & 8.6 & $<0.001$ & $\mathrm{~m}$ \\
\hline Vitamin D status & $\leq 20$ & $39(48.8 \%)$ & & $64(92.8 \%)$ & & $<0.001$ & $\mathrm{X}^{2}$ \\
\hline & $21-29$ & $29(6.3 \%)$ & & $5(7.2 \%)$ & & & \\
\hline & $\geq 30$ & $12(15.0 \%)$ & & $0(0.0 \%)$ & & & \\
\hline
\end{tabular}

m Mann-Whitney U test / I Independent Samples t-test/ $\mathrm{X}^{2}$ Chi-Squared test

Vitamin D has important immune functions. Considering in a context of a viral infection, the beneficial anti-viral effect of vitamin D can be grouped into three categories: enhancing physical barrier against the viruses and beneficial effects on innate and adaptive immunity $(15,16)$. Vitamin D supports the innate immune system by means of inducing the secretion of several antimicrobial peptides such as defensins. In addition, the active form of vitamin D, calcitriol, has itself several virus suppressive effects. Cytokine storm was found to play an important role in the rapid deterioration of some patients with COVID-19 (17). Importantly, vitamin D can limit the devastating effects of cytokine storm via reducing the release of proinflammatory Th1 derived cytokines such as TNF-alpha and interferon-gamma $(18,19)$. Last but not least, vitamin D also has an important favorable impact on the adaptive immune system, particularly through the actions of calcitriol. Calcitriol stimulates $T$ regulatory lymphocytes and suppresses cytokine release both from Th1 and Th2 lymphocytes $(20,21)$.

COVID-19 loaded a proportionately heavy burden on the elderly $(22,23)$. One of the main reasons for this was the common occurrence of chronic medical conditions in this age group. Moreover, both the presence of comorbidities and 


\section{THE JOURNAL OF NUTRITION, HEALTH \& AGING}

Table 4

Univariate and multivariate logistic regression analysis showing independent predictors of in-hospital mortality

\begin{tabular}{|c|c|c|c|c|c|c|}
\hline & \multicolumn{6}{|c|}{ Logistic regression analysis } \\
\hline & \multicolumn{3}{|c|}{ Univariate analysis } & \multicolumn{3}{|c|}{ Multivariate analysis } \\
\hline & OR & $95 \% \mathrm{CI}$ & P-value & OR & $95 \% \mathrm{CI}$ & P-value \\
\hline Age & 1.036 & $1.012-.060$ & 0.003 & & & \\
\hline Smoking & 2.161 & $1.085-4.304$ & 0.028 & & & \\
\hline Hyperlipidemia & 3.116 & $1.446-6.717$ & 0.004 & & & \\
\hline Diabetes mellitus & 2.400 & $1.230-4.682$ & 0.010 & & & \\
\hline Chronic kidney disease & 3.220 & $1.353-7.661$ & 0.008 & & & \\
\hline Chronic atrial fibrillation & 9.054 & $1.965-41.718$ & 0.005 & & & \\
\hline Congestive heart failure & 4.836 & $1.510-15.491$ & 0.008 & & & \\
\hline Acute kidney injury & 4.000 & $1.226-13.051$ & 0.022 & & & \\
\hline eGFR & 0.979 & $0.968-0.989$ & $<0.001$ & & & \\
\hline Hemoglobin & 0.822 & $0.699-0.968$ & 0.018 & & & \\
\hline Neutrophil count & 1.408 & $1.226-1.617$ & $<0.001$ & & & \\
\hline Lymphocyte count & 1.743 & $1.419-2.142$ & $<0.001$ & 1.375 & $1.092-1.731$ & 0.007 \\
\hline White blood cell count & 0.640 & $0.551-0.744$ & $<0.001$ & 0.815 & $0.679-0.979$ & 0.029 \\
\hline C-reactive protein & 1.008 & $1.004-1.013$ & 0.001 & & & \\
\hline Albumin & 0.158 & $0.071-0.353$ & $<0.001$ & 0.196 & $0.067-0.572$ & 0.003 \\
\hline Calcium & 0.267 & $0.141-0.506$ & $<0.001$ & & & \\
\hline $25(\mathrm{OH})$ vitamin $\mathrm{D}$ & 0.896 & $0.858-0.936$ & $<0.001$ & 0.927 & $0.875-0.982$ & 0.010 \\
\hline
\end{tabular}

Logistic Regression (Forward LR), CI: Confidence interval OR: Odds ratio

advancing age per se are associated with reduced $25(\mathrm{OH})$ vitamin D levels (24). We should take the effects of the lockdown and avoidance of sun exposure into account on the already insufficient $25(\mathrm{OH})$ vitamin D levels.

Hypothesis regarding the potential deleterious roles of vitamin D deficiency on COVID-19 patients initially originated from the studies in which $25(\mathrm{OH})$ vitamin $\mathrm{D}$ was shown to be related to increased susceptibility to infection $(25,26)$. However, some studies did not show the beneficial effects of vitamin D supplementation on the prevention and treatment of viral respiratory infections $(27,28)$. Then, some authors reported that the mean level of $25(\mathrm{OH})$ vitamin $\mathrm{D}$ level was inversely correlated with COVID-19 infection rates and mortality in 20 European countries (5). The authors emphasized the possible association of severely deficient vitamin D status and very high case fatality rates among the elderly in Spain and Italy. Some others also speculated that COVID-19 related mortality was lower in countries south of Latitude 35 degrees north might be related to vitamin D deficiency that is more prevalent due to limited sun exposure (29).

Despite all the experimental evidence regarding the antiviral and immunity supporting roles of vitamin $\mathrm{D}$, along with indirect clinical evidence showing the potential detrimental effect of vitamin D deficiency on upper respiratory viral infections, to our knowledge, only a few studies evaluated the vitamin D and mortality association in COVID-19 patients. Daneshkhah et al. (30) reported that in COVID-19 patients, serum C-reactive protein levels were inversely correlated with serum $25(\mathrm{OH})$ $\mathrm{D}$ levels. The authors concluded that since CRP is a marker of inflammation, vitamin D deficiency might be related to increased inflammation and cytokine storm in these patients. Alipio first reported the independent association between vitamin D deficiency and increased mortality (31). In another retrospective study, Raharusun and colleagues showed that $25(\mathrm{OH})$ vitamin $\mathrm{D}$ value was an independent predictor of mortality.

Our results present important data regarding the relationship between vitamin D deficiency and prognosis of COVID-19. First of all, the prevalence of vitamin D deficiency/insufficiency was very high among hospitalized moderate-to-severely ill COVID-19 patients. The mean level of $25(\mathrm{OH})$ vitamin D was $15.2 \pm 10.3 \mathrm{ng} / \mathrm{mL}$. Moreover, as COVID-19 severity increased, the level of $25(\mathrm{OH})$ vitamin D further reduced. Multivariable logistic regression analysis revealed that $25(\mathrm{OH})$ vitamin D level was independently associated with mortality, even after controlling for age and comorbid diseases was performed. Serum 25(OH) vitamin D level was positively correlated with serum C-reactive protein levels and negatively correlated with 


\section{VITAMIN D AND MORTALITY IN COVID-19 IN TURKEY}

both neutrophil and lymphocyte counts. Thus, the unfavorable effects of low serum vitamin D levels in terms of disease severity and mortality might be partly due to the increased inflammation. Our results also showed that mean serum $25(\mathrm{OH})$ vitamin $\mathrm{D}$ level was negatively correlated with increasing age $(\mathrm{r}=-0.3, \mathrm{p}<0.001)$. Thus, we speculate that disproportionately unfavorable clinical outcomes seen in the elderly may be, in part, due to the age-related vitamin D deficiency.

Some limitations of the present study deserve mention. First, since this study was a retrospective analysis, we did not control all the factors that potentially might have impacted the mortality rate. Our sample size is relatively small. However, it should be kept in mind that we only included patients who have moderateto-severe COVID-19.

\section{Conclusion}

In conclusion, our analysis revealed that vitamin D insufficiency/deficiency was quite prevalent among hospitalized COVID-19 patients. The level of $25(\mathrm{OH})$ vitamin D level was inversely related to the severity of the COVID-19. Moreover, vitamin D level was an independent predictor of COVID-19 related mortality. Since it is still rampant in many areas of the world, and considering the lack of an effective vaccine and specific drugs, measures to strengthen the immune system might be strongly considered. Before recommending the routine application of high-dose vitamin D in vulnerable populations, controlled studies investigating the effects of vitamin D supplementation on the COVID-19 severity and mortality are warranted.

Conflict of interest: The authors declare that they have no conflict of interest.

Ethical standards: The study protocol was approved by the Hospital Clinical Studies Ethical Committee (2020.06.1.01.072 and 12 June 2020).

\section{References}

1. Johns Hopkins University Coronavirus Resource Center, https://coronavirus.jhu.edu/ map.html, accessed 14th June, 2020.

2. Guan WJ, Liang WH, Zhao Y, Liang HR, Chen ZS, Li YM, et al. Comorbidity and its impact on 1590 patients with COVID-19 in China: a nationwide analysis. Eur Respir J. 2020;55(5).

3. Wang B, Li R, Lu Z, Huang Y. Does comorbidity increase the risk of patients with COVID-19: evidence from meta-analysis. Aging (Albany NY). 2020;12(7):6049-57.

4. Rhodes JM, Subramanian S, Laird E, Kenny RA. Editorial: low population mortality from COVID-19 in countries south of latitude 35 degrees North supports vitamin D as a factor determining severity. Aliment Pharmacol Ther. 2020;51(12):1434-7.

5. Ilie PC, Stefanescu S, Smith L. The role of vitamin D in the prevention of coronavirus disease 2019 infection and mortality. Aging Clin Exp Res. 2020.

6. Kara M, Ekiz T, Ricci V, Kara O, Chang KV, Ozcakar L. 'Scientific Strabismus' or Two Related Pandemics: COVID-19 \& Vitamin D Deficiency. Br J Nutr. 2020:1-20.

7. Aranow C. Vitamin D and the immune system. J Investig Med. 2011;59(6):881-6.

8. Teymoori-Rad M, Shokri F, Salimi V, Marashi SM. The interplay between vitamin D and viral infections. Rev Med Virol. 2019;29(2):e2032.

9. Martineau AR, Jolliffe DA, Hooper RL, Greenberg L, Aloia JF, Bergman P, et al Vitamin D supplementation to prevent acute respiratory tract infections: systematic review and meta-analysis of individual participant data. BMJ. 2017·356:i6583.

10. D'Avolio A, Avataneo V, Manca A, Cusato J, De Nicolo A, Lucchini R, et al 25-Hydroxyvitamin D Concentrations Are Lower in Patients with Positive PCR for SARS-CoV-2. Nutrients. 2020;12(5)

11. Hastie CE, Mackay DF, Ho F, Celis-Morales CA, Katikireddi SV, Niedzwiedz CL, et al. Vitamin D concentrations and COVID-19 infection in UK Biobank. Diabetes Metab Syndr. 2020;14(4):561-5.

12. Raharusun, Prabowo and Priambada, Sadiah and Budiarti, Cahni and Agung, Erdie and Budi, Cipta, Patterns of COVID-19 Mortality and Vitamin D: An Indonesian Study (April 26, 2020). Available at SSRN: https://ssrn.com/abstract=3585561 or http://dx.doi.org/10.2139/ssrn.3585561.

13. Holick MF. Vitamin D status: measurement, interpretation, and clinical application. Ann Epidemiol. 2009;19(2):73-8.

14. Diagnosis and Treatment Protocol for Novel Coronavirus Pneumonia (Trial Version 7). Chin Med J (Engl). 2020;133(9):1087-95.

15. Grant WB, Lahore H, McDonnell SL, Baggerly CA, French CB, Aliano JL, et al Evidence that Vitamin D Supplementation Could Reduce Risk of Influenza and COVID-19 Infections and Deaths. Nutrients. 2020;12(4).

16. Rondanelli M, Miccono A, Lamburghini S, Avanzato I, Riva A, Allegrini P, et al. Self-Care for Common Colds: The Pivotal Role of Vitamin D, Vitamin C, Zinc, and Echinacea in Three Main Immune Interactive Clusters (Physical Barriers, Innate and Adaptive Immunity) Involved during an Episode of Common Colds-Practical Advice on Dosages and on the Time to Take These Nutrients/Botanicals in order to Prevent or Treat Common Colds. Evid Based Complement Alternat Med. 2018;2018:5813095.

17. Ye Q, Wang B, Mao J. The pathogenesis and treatment of the 'Cytokine Storm' in COVID-19. J Infect. 2020;80(6):607-13.

18. Sharifi A, Vahedi H, Nedjat S, Rafiei H, Hosseinzadeh-Attar MJ. Effect of singledose injection of vitamin D on immune cytokines in ulcerative colitis patients: a randomized placebo-controlled trial. APMIS. 2019;127(10):681-7.

19. Aygun H. Vitamin D can prevent COVID-19 infection-induced multiple organ damage. Naunyn Schmiedebergs Arch Pharmacol. 2020.

20. Jeffery LE, Burke F, Mura M, Zheng Y, Qureshi OS, Hewison M, et al 1,25-Dihydroxyvitamin D3 and IL-2 combine to inhibit $\mathrm{T}$ cell production of inflammatory cytokines and promote development of regulatory $\mathrm{T}$ cells expressing CTLA-4 and FoxP3. J Immunol. 2009;183(9):5458-67.

21. Cantorna MT, Snyder L, Lin YD, Yang L. Vitamin D and 1,25(OH)2D regulation of T cells. Nutrients. 2015;7(4):3011-21.

22. Liu K, Chen Y, Lin R, Han K. Clinical features of COVID-19 in elderly patients: A comparison with young and middle-aged patients. J Infect. 2020;80(6):e14-e8.

23. Zheng Z, Peng F, Xu B, Zhao J, Liu H, Peng J, et al. Risk factors of critical \& mortal COVID-19 cases: A systematic literature review and meta-analysis. J Infect. 2020.

24. Kaur J, Ferguson SL, Freitas E, Miller R, Bemben D, Knehans A, et al. Association of Vitamin D Status with Chronic Disease Risk Factors and Cognitive Dysfunction in 50(-)70 Year Old Adults. Nutrients. 2019;11(1).

25. Arihiro S, Nakashima A, Matsuoka M, Suto S, Uchiyama K, Kato T, et al Randomized Trial of Vitamin D Supplementation to Prevent Seasonal Influenza and Upper Respiratory Infection in Patients With Inflammatory Bowel Disease. Inflamm Bowel Dis. 2019;25(6):1088-95.

26. Cannell JJ, Vieth R, Umhau JC, Holick MF, Grant WB, Madronich S, et al. Epidemic influenza and vitamin D. Epidemiol Infect. 2006;134(6):1129-40.

27. Aglipay M, Birken CS, Parkin PC, Loeb MB, Thorpe K, Chen Y, et al. Effect of High-Dose vs Standard-Dose Wintertime Vitamin D Supplementation on Viral Upper Respiratory Tract Infections in Young Healthy Children. JAMA. 2017;318(3):245-54.

28. Hueniken K, Aglipay M, Birken CS, Parkin PC, Loeb MB, Thorpe KE, et al. Effect of High-Dose Vitamin D Supplementation on Upper Respiratory Tract Infection Symptom Severity in Healthy Children. Pediatr Infect Dis J. 2019;38(6):564-8.

29. Marik PE, Kory P, Varon J. Does vitamin D status impact mortality from SARS CoV-2 infection? Med Drug Discov. 2020:100041

30. Daneshkhah A, Agrawal V, Eshein A, Subramanian H, Roy HK, Backman V. The Possible Role of Vitamin D in Suppressing Cytokine Storm and Associated Mortality in COVID-19 Patients. medRxiv. 2020:2020.04.08.20058578.

31. Alipio, Mark, Vitamin D Supplementation Could Possibly Improve Clinical Outcomes of Patients Infected with Coronavirus-2019 (COVID-19) (April 9, 2020). Available a SSRN: https://ssrn.com/abstract=3571484 or http://dx.doi.org/10.2139/ssrn.3571484 . 